\title{
Solitary Wave Solutions of Nonlinear Conformable Time-Fractional Boussinesq Equations
}

\author{
O. Guner ${ }^{a, *}$, A. BeKIR ${ }^{b}$ And K. Hosseini ${ }^{c}$ \\ ${ }^{a}$ Cankiri Karatekin University, Department of International Trade, Cankiri, Turkey \\ ${ }^{b}$ Neighbourhood of Akcaglan, Imarli Str. Number: 28/4, 26030, Eskisehir, Turkey \\ ${ }^{c}$ Islamic Azad University, Department of Mathematics, Rasht Branch, Rasht, Iran \\ (Received September 22, 2018; revised version April 3, 2019; in final form May 14, 2019)
}

\begin{abstract}
In this paper, the $\left(\frac{G^{\prime}}{G}\right)$-expansion method based on conformable fractional derivative is proposed to solve time fractional partial differential equations in mathematical physics. To illustrate the validity of this method, we solve the Boussinesq equation, coupled time-fractional Boussinesq equations and a variety of exact solutions for them are successfully established. With the help of Maple software, three-dimensional solution graphs are presented.
\end{abstract}

DOI: 10.12693/APhysPolA.136.135

PACS/topics: conformable fractional derivative, time-fractional Boussinesq equations, $\left(\frac{G^{\prime}}{G}\right)$-expansion method

\section{Introduction}

In recent years, fractional differential equations (FDEs) which are the generalization of differential equations are successful models of real life problems and have many applications in various fields in science. For example, the use of FDEs is seen in the fields of physics, engineering, systems identification, fluid flow, control problem, fluid mechanics, mathematical biology, signal processing, heat diffusion, polymers, controller tuning, digital circuit synthesis, and viscoelastic materials [1-6]. Also some applications of nonlinear FDEs can be found in economics and finances [7-9].

There are many methods for finding the approximate solutions for nonlinear FDEs such as variation iteration method, homotopy perturbation method, Adomian decomposition method, differential transform method, and homotopy analysis method [10-20]. However, an effective and general method for solving these types of equations cannot be found. In particular, the exact solution of FDEs was very limited until $\mathrm{Li}$ and $\mathrm{He}$ found the fractional complex transformation. After this transform, various analytical methods were proposed such as the fractional sub-equation method, the functional variable method, the $\left(G^{\prime} / G\right)$-expansion method, the expfunction method, the first integral method, the modified trial equation method, the modified simple equation method, the ansatz method, the exponential rational function method, the $\left(G^{\prime} / G-1 / G\right)$-expansion method, and the modified Kudryashov method [21-34].

The Boussinesq-type equations are very commonly used in nonlinear fractional temporal evolution formulated for describing the dynamics of water with small amplitude and long wave. In coastal areas, Boussinesq-

*corresponding author; e-mail: ozkanguner@karatekin.edu.tr type equations are also the most important equations in the prediction of wave transformations. In coastal and ocean engineering this equation is widely used. Also, the tsunami wave modeling and mathematical modeling of tidal oscillations show some of the applications of fractional Boussinesq equation in ocean engineering [35-40].

Many studies were done on FDEs with different derivatives such as Weyl and Chen, Cresson, Grunwald-Letnikov, Riemann-Liouville, Caputo, modified Riemann-Liouville derivatives [41-44]. However, new derivatives should be proposed in order to deal better with the real world problems. Recently, the conformable fractional derivative was proposed by Khalil et al. [45], which can rectify the shortcomings of the previous definitions. Herein, definition and some properties of the conformable fractional derivative are presented [4648]. One can see many useful studies related to this new fractional derivative definition in [49-52]. In Ref. [53], physical and geometrical interpretations of this new derivative which thus indicate potential applications in physics and engineering are given. Also, principles for the definition of local fractional definition have been discussed [54-56]:

$$
T_{\alpha}(f(t))=\lim _{\xi \rightarrow 0} \frac{f\left(t+\xi t^{1-\alpha}\right)-f(t)}{\xi},
$$

in which $f:[0, \infty) \rightarrow R, t>0$ and $0<\alpha<1$. Some properties:

Property 1:

$$
T_{\alpha}\left(C_{1} f(t)+C_{2} g(t)\right)=C_{1} T_{\alpha}(f(t))+C_{2} T_{\alpha}(g(t)) \text {, }
$$

Property 2:

$$
T_{\alpha}\left(t^{\mu}\right)=\mu t^{\mu-\alpha},
$$

where $\forall C_{1}, C_{2}, \mu \in R$.

Property 3:

$$
T_{\alpha}(f(t) g(t))=g(t) T_{\alpha}(f(t))+f(t) T_{\alpha}(g(t)),
$$




\section{Property 4:}

$T_{\alpha}\left(\frac{f(t)}{g(t)}\right)=\frac{g(t) T_{\alpha}(f(t))-f(t) T_{\alpha}(g(t))}{g(t)^{2}}$,

\section{Property 5:}

$$
T_{\alpha}(C)=0 \text {, }
$$

where $C$ is constant.

The organization of this paper is as follows. In Sect. 2, we give theoretical background and preliminaries. In Sects. 3 and 4, we implement this method to establish exact solution for conformable time-fractional Boussinesq equation and coupled conformable time-fractional Boussinesq equations. Finally, in Sect. 5 a brief conclusion is provided.

\section{Theoretical background and preliminaries}

We consider the following general conformable time fractional partial differential equation of the type

$$
Q\left(u, D_{t}^{\alpha} u, u_{x}, D_{t}^{2 \alpha} u, u_{x x}, \ldots\right)=0, \quad 0<\alpha<1,
$$

where $u$ is an unknown function, $Q$ is a polynomial of $u$, and $D^{\alpha}$ are partial fractional derivatives of $u$.

We use the transformation

$$
\begin{aligned}
& u(x, t)=f(\theta), \\
& \theta=x-\frac{m t^{\alpha}}{\alpha},
\end{aligned}
$$

where $m$ is nonzero arbitrary constant. The transformation given in Eq. (8) is called as the fractional complex transform, proposed by a Chinese mathematician, Li [57]. Equation (7) can be turned into the following nonlinear ODE:

$$
H\left(f, f^{\prime}, f^{\prime \prime}, f^{\prime \prime \prime}, \ldots\right)=0 .
$$

where the " $f^{\prime \prime} "=\frac{\mathrm{d} f}{\mathrm{~d} \theta}$.

We look for its solution $f(\theta)$ in the polynomial form

$$
f(\theta)=\sum_{i=0}^{z} a_{i}\left(\frac{G^{\prime}}{G}\right)^{i}, \quad a_{z} \neq 0,
$$

where $a_{i}$ are constants, while $G(\theta)$ is the solution of the auxiliary linear second order ODE

$$
G^{\prime \prime}(\theta)+\lambda G^{\prime}(\theta)+\mu G(\theta)=0,
$$

with $\lambda$ and $\mu$ are being constants. $z$ is a positive integer which is determined by the homogeneous balancing method in Eq. (9). Substituting Eq. (10) into Eq. (9) with the help of Maple, we determine $a_{i}$ and $m$. When we substitute $a_{i}(i=0,1,2, \ldots, z), m$, and the solutions of Eq. (11) into Eq. (9), we can derive traveling wave solutions of Eq. (7) [58, 59].

\section{Conformable time-fractional Boussinesq equation}

We consider the following nonlinear conformable time-fractional Boussinesq Eq. [60]:

$$
D_{t}^{2 \alpha} u-u_{x x}+\left(u^{2}\right)_{x x}-u_{x x x x}=0, \quad 0<\alpha \leq 1,
$$

which describes the surface water waves whose horizontal scale is much larger than the depth of the water. Equation (12) may be used to describe the spread of wave in magnetic field. It is also an important nonlinear model arising in physics, hydromechanics, and optics. It can also be used to describe a series of physical aspects about the spread of wave in plasma and nonlinear wave [61-65].

By applying the traveling wave transformation (8) to this equation and integrating it twice, and then setting the constants of integration equal to zero, it can be turned into a nonlinear ODE

$$
f^{\prime \prime}+\left(m^{2}-1\right) f-f^{2}=0,
$$

where " $f^{\prime \prime}=\frac{\mathrm{d} f}{\mathrm{~d} \theta}$.

Balancing the highest derivative term $f^{\prime \prime}$ with the nonlinear term $f^{2}$ in Eq. (13) yields $z=2$. Hence, we can write the solution of Eq. (13) in the form

$$
f(\theta)=a_{0}+a_{1}\left(\frac{G^{\prime}}{G}\right)+a_{1}\left(\frac{G^{\prime}}{G}\right)^{2}, \quad a_{2} \neq 0 .
$$

Substituting Eq. (14) with Eq. (11) along with its necessary derivatives into Eq. (13), collecting all terms with the same powers of $\left(\frac{G^{\prime}}{G}\right)$, and setting them to zero we get the system of algebraic equations, and solving it by Maple gives

\section{Part A}

$$
\begin{aligned}
& a_{0}=6 \mu, \quad a_{1}=6 \lambda, \quad a_{2}=6, \\
& m=\mp \sqrt{1+4 \mu-\lambda^{2}},
\end{aligned}
$$

where $\lambda$ and $\mu$ are arbitrary constants.

By using Eq. (15), expression (14) can be written as

$$
f(\theta)=6 \mu+6 \lambda\left(\frac{G^{\prime}}{G}\right)+6\left(\frac{G^{\prime}}{G}\right)^{2} .
$$

From (16) and the general solutions of Eq. (11), we deduce the following traveling wave solutions:

When $\lambda^{2}-4 \mu>0$,

$$
\begin{aligned}
& f_{1,2}(\theta)=\frac{3\left(4 \mu-\lambda^{2}\right)}{2} \\
& \times\left[1-\left(\frac{C_{1} \sinh \frac{1}{2} \sqrt{\lambda^{2}-4 \mu} \theta+C_{2} \cosh \frac{1}{2} \sqrt{\lambda^{2}-4 \mu} \theta}{C_{1} \cosh \frac{1}{2} \sqrt{\lambda^{2}-4 \mu} \theta+C_{2} \sinh \frac{1}{2} \sqrt{\lambda^{2}-4 \mu} \theta}\right)^{2}\right],
\end{aligned}
$$

where $\theta=x \mp \frac{\sqrt{1+4 \mu-\lambda^{2}} t^{\alpha}}{\alpha}$.

When $\lambda^{2}-4 \mu<0$,

$$
\begin{gathered}
f_{3,4}(\theta)=\frac{3\left(4 \mu-\lambda^{2}\right)}{2} \\
\times\left[1+\left(\frac{-C_{1} \sin \frac{1}{2} \sqrt{4 \mu-\lambda^{2}} \theta+C_{2} \cos \frac{1}{2} \sqrt{4 \mu-\lambda^{2}} \theta}{C_{1} \cos \frac{1}{2} \sqrt{4 \mu-\lambda^{2}} \theta+C_{2} \sin \frac{1}{2} \sqrt{4 \mu-\lambda^{2}} \theta}\right)^{2}\right],
\end{gathered}
$$

where $\theta=x \mp \frac{\sqrt{1+4 \mu-\lambda^{2}} t^{\alpha}}{\alpha}$.

When $\lambda^{2}-4 \mu=0$,

$$
u_{1,2}(x, t)=6\left(\frac{C_{1}}{C_{2}+C_{1}\left(x \mp \frac{t^{\alpha}}{\alpha}\right)}\right)^{2} \text {. }
$$


In particular, if $C_{1} \neq 0, C_{2}=0, \lambda>0, \mu=0$, then $f_{1,2}$ and $f_{3,4}$ become

$$
u_{3,4}(x, t)=-\frac{3 \lambda^{2}}{2} \operatorname{sech}^{2}\left(\frac{\lambda x}{2} \mp \frac{\lambda \sqrt{1-\lambda^{2}} t^{\alpha}}{2 \alpha}\right) .
$$

\section{Part B}

$$
\begin{aligned}
& a_{0}=\lambda^{2}+2 \mu, \quad a_{1}=6 \lambda, \quad a_{2}=6, \\
& m=\mp \sqrt{\lambda^{2}-4 \mu+1},
\end{aligned}
$$

where $\lambda$ and $\mu$ are arbitrary constants.

By using Eq. (21), expression (14) can be written as

$$
f(\theta)=\lambda^{2}+2 \mu+6 \lambda\left(\frac{G^{\prime}}{G}\right)+6\left(\frac{G^{\prime}}{G}\right)^{2} .
$$

From (22) and the general solutions of Eq. (11), we deduce the following traveling wave solutions:

When $\lambda^{2}-4 \mu>0$,

$$
\begin{aligned}
& f_{5,6}(\theta)=\frac{4 \mu-\lambda^{2}}{2} \\
& \times\left[1-3\left(\frac{C_{1} \sinh \frac{1}{2} \sqrt{\lambda^{2}-4 \mu} \theta+C_{2} \cosh \frac{1}{2} \sqrt{\lambda^{2}-4 \mu} \theta}{C_{1} \cosh \frac{1}{2} \sqrt{\lambda^{2}-4 \mu} \theta+C_{2} \sinh \frac{1}{2} \sqrt{\lambda^{2}-4 \mu} \theta}\right)^{2}\right],
\end{aligned}
$$

where $\theta=x \mp \frac{\sqrt{\lambda^{2}-4 \mu+1} t^{\alpha}}{\alpha}$.

When $\lambda^{2}-4 \mu<0$,

$f_{7,8}(\theta)=\frac{4 \mu-\lambda^{2}}{2}$

$$
\times\left[1+3\left(\frac{-C_{1} \sin \frac{1}{2} \sqrt{4 \mu-\lambda^{2}} \theta+C_{2} \cos \frac{1}{2} \sqrt{4 \mu-\lambda^{2}} \theta}{C_{1} \cos \frac{1}{2} \sqrt{4 \mu-\lambda^{2}} \theta+C_{2} \sin \frac{1}{2} \sqrt{4 \mu-\lambda^{2}} \theta}\right)^{2}\right],
$$

where $\theta=x \mp \frac{\sqrt{\lambda^{2}-4 \mu+1} t^{\alpha}}{\alpha}$.

When $\lambda^{2}-4 \mu=0$, we get solution (3.8). When we take $C_{1} \neq 0, C_{2}=0, \lambda>0, \mu=0$, then $f_{5,6}$ and $f_{7,8}$ become

$$
\begin{aligned}
& u_{1}(x, t)= \\
& \frac{\lambda^{2}}{2}\left[-1+3 \tanh ^{2}\left(\frac{\lambda x}{2} \mp \frac{\lambda \sqrt{\lambda^{2}+1} t^{\alpha}}{2 \alpha}\right)\right] .
\end{aligned}
$$

\section{Coupled conformable time-fractional Boussinesq equations}

Secondly, we consider the coupled conformable timefractional Boussinesq equations [66]:

$$
\begin{aligned}
& D_{t}^{\alpha} u-v_{x}=0, \\
& D_{t}^{\alpha} v+b\left(u^{2}\right)_{x}-a u_{x x x}=0,
\end{aligned}
$$

where $0<\alpha \leq 1$ and this is used to model the two-way propagation of certain water waves in a uniform horizontal channel filled with an irrotational and inviscid liquid.
Similarly, by introducing the traveling wave transformation

$$
\begin{aligned}
& u(x, t)=f(\theta), \quad \theta=x-\frac{m t^{\alpha}}{\alpha}, \\
& v(x, t)=g(\theta), \quad \theta=x-\frac{m t^{\alpha}}{\alpha},
\end{aligned}
$$

Equation (26) changes into a system of ODE as

$$
\begin{aligned}
& -m f^{\prime}-g^{\prime}=0, \\
& -m g^{\prime}+b\left(f^{2}\right)^{\prime}-a f^{\prime \prime \prime}=0,
\end{aligned}
$$

where " $f^{\prime} "=\frac{\mathrm{d} f}{\mathrm{~d} \theta}$ and " $g$ " $=\frac{\mathrm{d} g}{\mathrm{~d} \theta}$. Integrating (28) once and taking the constants of integration equal to zero, we get

$$
\begin{aligned}
& g=-m f, \\
& -m g+b f^{2}-a f^{\prime \prime}=0 .
\end{aligned}
$$

Inserting (29) into (30), gives

$$
m^{2} f+b f^{2}-a f^{\prime \prime}=0 \text {. }
$$

Similarly, we can determine value of $z$ by balancing $f^{2}$ and $f^{\prime \prime}$ in Eq. (31) and we get $z=2$. We can suppose that the solution of Eq. (31) is of the form

$$
f(\theta)=a_{0}+a_{1}\left(\frac{G^{\prime}}{G}\right)+a_{2}\left(\frac{G^{\prime}}{G}\right)^{2}, \quad a_{2} \neq 0 .
$$

With similar approach as in the previous example we get

\section{Part A}

$$
\begin{aligned}
& a_{0}=\frac{6 a \mu}{b}, \quad a_{1}=\frac{6 a \lambda}{b}, \quad a_{2}=\frac{6 a}{b}, \\
& m=\mp \sqrt{a\left(\lambda^{2}-4 \mu\right)},
\end{aligned}
$$

\section{Part B}

$$
\begin{aligned}
& a_{0}=\frac{a\left(\lambda^{2}+2 \mu\right)}{b}, \quad a_{1}=\frac{6 a \lambda}{b}, \quad a_{2}=\frac{6 a}{b}, \\
& m=\mp \sqrt{a\left(4 \mu-\lambda^{2}\right)},
\end{aligned}
$$

where $\lambda$ and $\mu$ are arbitrary constants. By using Eq. (33), expression (32) can be written as

$$
f(\theta)=\frac{6 a \mu}{b}+\frac{6 a \lambda}{b}\left(\frac{G^{\prime}}{G}\right)+\frac{6 a}{b}\left(\frac{G^{\prime}}{G}\right)^{2} .
$$

Using the general solutions of Eq. (11), we obtain travelling wave solutions of Eqs. (26) as follows:

\section{Part A}

When $\lambda^{2}-4 \mu>0$,

$$
\begin{aligned}
& f_{1,2}(\theta)=\frac{3 a\left(4 \mu-\lambda^{2}\right)}{2 b} \\
& \times\left[1-\left(\frac{C_{1} \sinh \frac{1}{2} \sqrt{\lambda^{2}-4 \mu} \theta+C_{2} \cosh \frac{1}{2} \sqrt{\lambda^{2}-4 \mu} \theta}{C_{1} \cosh \frac{1}{2} \sqrt{\lambda^{2}-4 \mu} \theta+C_{2} \sinh \frac{1}{2} \sqrt{\lambda^{2}-4 \mu} \theta}\right)^{2}\right],
\end{aligned}
$$




$$
\begin{aligned}
& g_{1,2}(\theta)=-\frac{3 m a\left(4 \mu-\lambda^{2}\right)}{2 b} \\
& \times\left[1-\left(\frac{C_{1} \sinh \frac{1}{2} \sqrt{\lambda^{2}-4 \mu} \theta+C_{2} \cosh \frac{1}{2} \sqrt{\lambda^{2}-4 \mu} \theta}{C_{1} \cosh \frac{1}{2} \sqrt{\lambda^{2}-4 \mu} \theta+C_{2} \sinh \frac{1}{2} \sqrt{\lambda^{2}-4 \mu} \theta}\right)^{2}\right],
\end{aligned}
$$

where $\theta=x \mp \frac{\sqrt{a\left(\lambda^{2}-4 \mu\right)} t^{\alpha}}{\alpha}$.

When $\lambda^{2}-4 \mu<0$,

$$
\begin{aligned}
& f_{3,4}(\theta)=\frac{3 a\left(4 \mu-\lambda^{2}\right)}{2 b} \\
& \times\left[1+\left(\frac{-C_{1} \sin \frac{1}{2} \sqrt{4 \mu-\lambda^{2}} \theta+C_{2} \cos \frac{1}{2} \sqrt{4 \mu-\lambda^{2}} \theta}{C_{1} \cos \frac{1}{2} \sqrt{4 \mu-\lambda^{2}} \theta+C_{2} \sin \frac{1}{2} \sqrt{4 \mu-\lambda^{2}} \theta}\right)^{2}\right], \\
& g_{3,4}(\theta)=-\frac{3 m a\left(4 \mu-\lambda^{2}\right)}{2 b} \\
& \times\left[1+\left(\frac{C_{1} \sinh \frac{1}{2} \sqrt{\lambda^{2}-4 \mu} \theta+C_{2} \cosh \frac{1}{2} \sqrt{\lambda^{2}-4 \mu} \theta}{C_{1} \cosh \frac{1}{2} \sqrt{\lambda^{2}-4 \mu} \theta+C_{2} \sinh \frac{1}{2} \sqrt{\lambda^{2}-4 \mu} \theta}\right)^{2}\right],
\end{aligned}
$$

where $\theta=x \mp \frac{\sqrt{a\left(\lambda^{2}-4 \mu\right)} t^{\alpha}}{\alpha}$.

In particular, if $C_{1} \neq 0, C_{2}=0, \lambda>0, \mu=0$, then $f_{1,2}, g_{1,2}$ and $f_{3,4}, g_{3,4}$ become

$$
\begin{aligned}
& u_{1,2}(x, t)=-\frac{3 a \lambda^{2}}{2 b} \operatorname{sech}^{2}\left(\frac{\lambda x}{2} \mp \frac{\lambda^{2} \sqrt{a} t^{\alpha}}{2 \alpha}\right) . \\
& v_{1,2}(x, t)=\frac{3 m a \lambda^{2}}{2 b} \operatorname{sech}^{2}\left(\frac{\lambda x}{2} \mp \frac{\lambda^{2} \sqrt{a} t^{\alpha}}{2 \alpha}\right) .
\end{aligned}
$$

\section{Part B}

By using Eq. (34), expression (32) can be written as

$$
f(\theta)=\frac{a\left(\lambda^{2}+2 \mu\right)}{b}+\frac{6 a \lambda}{b}\left(\frac{G^{\prime}}{G}\right)+\frac{6 a}{b}\left(\frac{G^{\prime}}{G}\right)^{2} .
$$

Using the general solutions of Eq. (11), we obtain travelling wave solutions of Eqs. (26) as follows:

When $\lambda^{2}-4 \mu>0$,

$$
\begin{aligned}
& f_{1,2}(\theta)=\frac{a\left(4 \mu-\lambda^{2}\right)}{2 b} \\
& \times\left[1-3\left(\frac{C_{1} \sinh \frac{1}{2} \sqrt{\lambda^{2}-4 \mu} \theta+C_{2} \cosh \frac{1}{2} \sqrt{\lambda^{2}-4 \mu} \theta}{C_{1} \cosh \frac{1}{2} \sqrt{\lambda^{2}-4 \mu} \theta+C_{2} \sinh \frac{1}{2} \sqrt{\lambda^{2}-4 \mu} \theta}\right)^{2}\right] \\
& g_{1,2}(\theta)=-\frac{m a\left(4 \mu-\lambda^{2}\right)}{2 b} \\
& \times\left[1-3\left(\frac{C_{1} \sinh \frac{1}{2} \sqrt{\lambda^{2}-4 \mu} \theta+C_{2} \cosh \frac{1}{2} \sqrt{\lambda^{2}-4 \mu} \theta}{C_{1} \cosh \frac{1}{2} \sqrt{\lambda^{2}-4 \mu} \theta+C_{2} \sinh \frac{1}{2} \sqrt{\lambda^{2}-4 \mu} \theta}\right)^{2}\right]
\end{aligned}
$$

where $\theta=x \mp \frac{\sqrt{a\left(4 \mu-\lambda^{2}\right)} t^{\alpha}}{\alpha}$.
When $\lambda^{2}-4 \mu<0$,

$$
\begin{aligned}
& f_{3,4}(\theta)=\frac{a\left(4 \mu-\lambda^{2}\right)}{2 b} \\
& \times\left[1+3\left(\frac{-C_{1} \sin \frac{1}{2} \sqrt{4 \mu-\lambda^{2}} \theta+C_{2} \cos \frac{1}{2} \sqrt{4 \mu-\lambda^{2}} \theta}{C_{1} \cos \frac{1}{2} \sqrt{4 \mu-\lambda^{2}} \theta+C_{2} \sin \frac{1}{2} \sqrt{4 \mu-\lambda^{2}} \theta}\right)^{2}\right] \\
& g_{3,4}(\theta)=-\frac{m a\left(4 \mu-\lambda^{2}\right)}{2 b} \\
& \times\left[1+3\left(\frac{C_{1} \sinh \frac{1}{2} \sqrt{\lambda^{2}-4 \mu} \theta+C_{2} \cosh \frac{1}{2} \sqrt{\lambda^{2}-4 \mu} \theta}{C_{1} \cosh \frac{1}{2} \sqrt{\lambda^{2}-4 \mu} \theta+C_{2} \sinh \frac{1}{2} \sqrt{\lambda^{2}-4 \mu} \theta}\right)^{2}\right],
\end{aligned}
$$

where $\theta=x \mp \frac{\sqrt{a\left(4 \mu-\lambda^{2}\right)} t^{\alpha}}{\alpha}$.

In particular, if $C_{1}^{\alpha} \neq 0, C_{2}=0, \lambda>0, \mu=0$, then $f_{1,2}, g_{1,2}$ and $f_{3,4}, g_{3,4}$ become

$$
\begin{aligned}
& u_{1,2}(x, t)=\frac{a \lambda^{2}}{2 b}\left[-1+3 \tanh ^{2}\left(\frac{\lambda x}{2} \mp \frac{\lambda^{2} \sqrt{-a} t^{\alpha}}{2 \alpha}\right)\right], \\
& v_{1,2}(x, t)=-\frac{m a \lambda^{2}}{2 b}\left[-1+3 \tanh ^{2}\left(\frac{\lambda x}{2} \mp \frac{\lambda^{2} \sqrt{-a} t^{\alpha}}{2 \alpha}\right)\right]
\end{aligned}
$$

\section{Results and discussion}

Exact solutions of Eqs. (19) and (20) have been used to draw the graphs as shown in Figs. 1 and 2 for classical and fractional order values of $\alpha$, within the interval $-100<x<100$ and $0<t<1$. Obtained exact solution (25) is described in Fig. 3, within the interval $-10<x<10$ and $0<t<1$. We plot the solutions of Eq. (38) for $u_{1,2}(x, t)$ in Fig. 4 within the interval $-100<x<100$ and $0<t<10$. Finally, in Fig. 5, we plot three dimensional graphics of $u_{1,2}(x, t)$ in Eq. (44) within the interval $-100<x<100$ and $0<t<10$. The obtained solutions in (19), (20), (25), (38), and (44) have not been reported in the literature before. These solutions, namely, rational, bright, and dark soliton solutions would be important for the explanation of some practical physical phenomena.
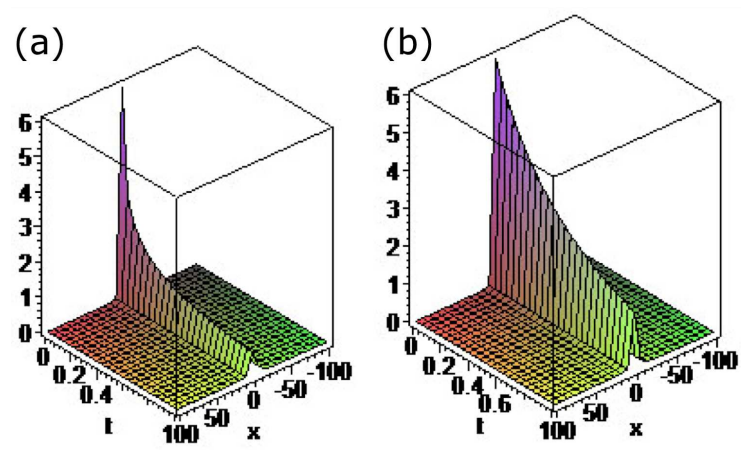

Fig. 1. Profiles of solution (19) with $\alpha=0.5$ (a) and $\alpha=1(\mathrm{~b})$, respectively, when $C_{1}=1, C_{2}=1$. 

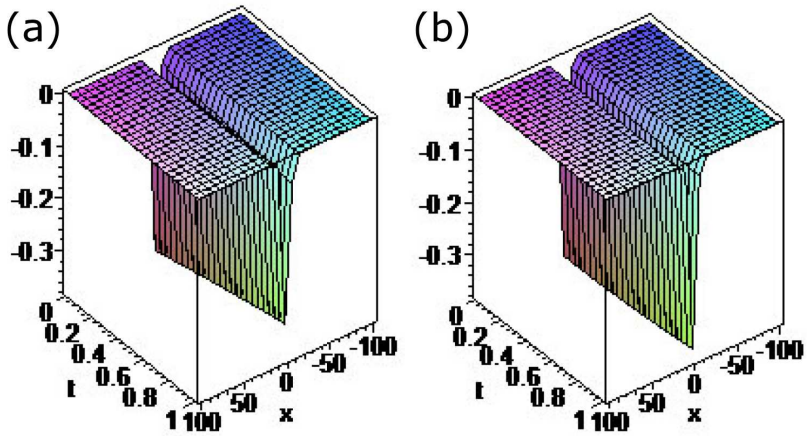

Fig. 2. Profiles of solution (20) with $\alpha=0.5$ (a) and $\alpha=1$ (b), respectively, when $\lambda=0.5$.
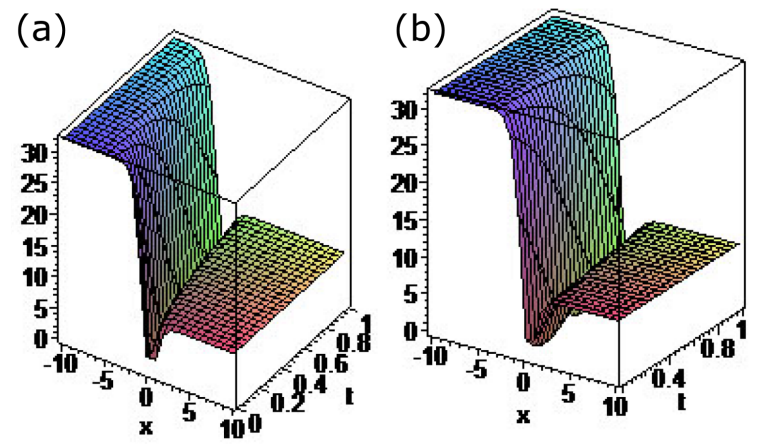

Fig. 3. Profiles of solution (25) with $\alpha=0.5$ (a) and $\alpha=1$ (b), respectively, when $\lambda=2$.
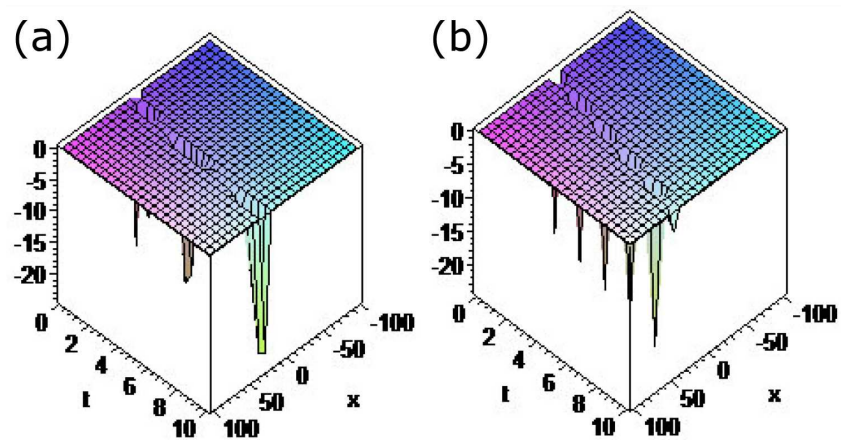

Fig. 4. Profiles of solution (38) for $u_{1,2}(x, t)$ with $\alpha=$ 0.5 (a) and $\alpha=1$ (b) respectively, when $a=4, b=1$, $\lambda=2$.

(a)

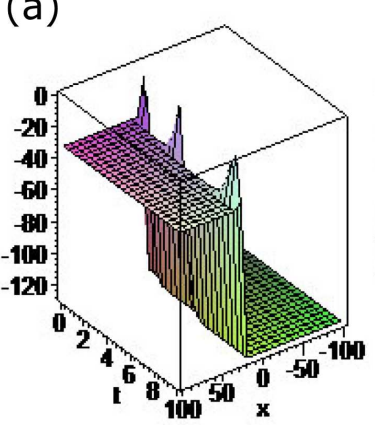

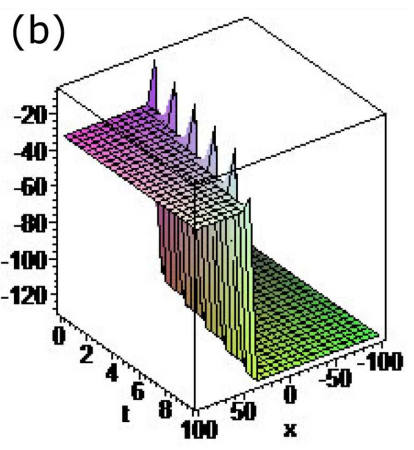

Fig. 5. Profiles of solution (44) for $u_{1,2}(x, t)$ with $\alpha=$ 0.5 (a) and $\alpha=1$ (b) respectively, when $a=-4, b=1$, $\lambda=2$.

The results show that the proposed method can also be applied to other fractional nonlinear FDEs with the conformable time fractional derivative. To better understand the physical interpretation, three-dimensional (3D) solution graphs are presented.

\section{Conclusion}

Using the $\left(G^{\prime} / G\right)$-expansion method, with the aid of software Maple, new variety of exact traveling wave solutions of the time fractional Boussinesq equation and coupled time-fractional Boussinesq equations are constructed. Fractional complex transform is proposed to convert the FDEs into ODEs, which is valid only for general"wave" solutions and it makes the solution procedure practical. As a result, we obtain three types of exact solutions for these equations: hyperbolic, trigonometric, and rational, including solitary waves and singular periodic solutions. Moreover, we obtain new exact solutions (19), (25), and (44) which are not established in the previous literature.

\section{References}

[1] D. Baleanu, K. Diethelm, E. Scalas, J.J. Trujillo, Nonlinearity and Chaos, World Sci., Boston 2012.

[2] I. Podlubny, Fractional Differential Equations, Academic Press, California 1999.

[3] A.A. Kilbas, H.M. Srivastava, J.J. Trujillo, Theory and Applications of Fractional Differential Equations, Elsevier, Amsterdam 2006.

[4] G. Samko, A.A. Kilbas, O. Marichev, Fractional Integrals and Derivatives: Theory and Applications, Gordon and Breach, Yverdon 1993.

[5] A. Bekir, O. Guner, A.C. Cevikel, Abstr. Appl. Anal. 2013, 426462 (2013).

[6] O. Guner, E. Aksoy, A. Bekir, A.C. Cevikel, Comput. Math. Appl. 71, 1259 (2016).

[7] I. Pan, S. Das, S. Das, Appl. Math. Modell. 39, 500 (2015).

[8] T. Skovranek, I. Podlubny, I. Petras, Econom. Modell. 29, 1322 (2012).

[9] J.A.T. Machado, M.E. Mata, Commun. Nonlin. Sci. Numer. Simulat. 22, 396 (2015).

[10] X.J. Yang, D. Baleanu, Y. Khan, S.T. Mohyud-Din, Rom. J. Phys. 59, 36 (2014).

[11] G.C Wu, D. Baleanu, Adv. Diff. Eq. 2013, 18 (2013).

[12] A.A. Elbeleze, A. Kılıçman, B.M. Taib, Math. Probl. Eng. 2013, 543848 (2013).

[13] N.A. Khan, A. Kara, A. Mahmood, Int. J. Num. Meth. Heat Fl. Flow 22, 175 (2012).

[14] J.H. He, Int. J. Mod. Phys. B 20, 1 (2006).

[15] K.A. Gepreel, Appl. Math. Lett. 24, 1428 (2011).

[16] V. Daftardar-Gejji, S. Bhalekar, Appl. Math. Comput. 202, 113 (2008).

[17] Z. Dahmani, M.M. Mesmoudi, R. Bebbiouchi, Electron. J. Qual. Theory Diff. Eq. 30, 1 (2008).

[18] M. Kurulay, M. Bayram, Commun. Nonlin. Sci. Numer. Simulat. 15, 1777 (2010). 
[19] A. El-Ajou, Z. Odibat, S. Momani, A. Alawneh, Int. J. Appl. Math. 40, 43 (2010).

[20] S.T. Mohyud-Din, A. Yıldırım, E. Yülüklü, Int. J. Num. Meth. Heat Fl. Flow 22, 928 (2012).

[21] S. Sahoo, S.S. Ray, Comput. Math. Appl. 70, 158 (2015).

[22] H. Jafari, J. Tajadodi, N. Kadkhoda, D. Baleanu, Abstr. Appl. Anal. 2013, 587179 (2013).

[23] W. Liu, K. Chen, Pramana J. Phys. 81, 377 (2013).

[24] A. Bekir, O. Guner, E. Aksoy, Appl. Comput. Math. 15, 88 (2016).

[25] M. Younis, Z. Zafar, K. Ul-Haq, M. Rahman, Am. J. Computat. Appl. Math. 3, 81 (2013).

[26] S. Sahoo, S.S. Ray, Nonlin. Dynam. 85, 1167 (2016).

[27] A. Bekir, O. Guner, A.H. Bhrawy, A. Biswas, Rom. J. Phys. 60, 360 (2015).

[28] O. Guner, A. Bekir, H. Bilgil, Adv. Nonlin. Anal. 4 201 (2015).

[29] A. Bekir, O. Guner, O. Unsal J. Computat. Nonlin. Dynam. 10, 021020 (2015).

[30] K. Khan, M.A. Akbar, J. Assoc. Arab Univ. Basic Appl. Sci. 15, 74 (2014).

[31] O. Guner, Chin. Phys. B 24, 100201 (2015).

[32] M. Mirzazadeh, Pramana J. Phys. 85, 17 (2015).

[33] E. Aksoy, M. Kaplan, A. Bekir, Waves Random Compl. Media 26, 142 (2016).

[34] H.G. Sun, W. Chen, Sci. China Ser. E 52, 680 (2009).

[35] A. Biswas, D. Milovic, A. Ranasinghe, Commun. Nonlinear Sci. Numer. Simulat. 14, 3738 (2009).

[36] G. Ebadi, S. Johnson, E. Zerrad, A. Biswas, J. King Saud University - Science 24, 237 (2012).

[37] E.V. Krishnan, S. Kumar, A. Biswas, Nonlin. Dynam. 70, 1213 (2012).

[38] A.J.M. Jawad, M.D. Petković, P. Laketa, A. Biswas, Sci. Iran. B 20, 179 (2013).

[39] H. Triki, A. Chowdhury, A. Biswas, U.P.B. Sci. Bull. Series A 75, 39 (2013).

[40] A. Biswas, M. Song, H. Triki, A.H. Kara, B.S. Ahmed, A. Strong, A. Hama, Appl. Math. Inf. Sci. 8, 949 (2014).

[41] W. Chen, H.G. Sun, Mod. Phys. Lett. B 23, 449 (2009).

[42] J. Cresson, J. Math. Phys 44, 4907 (2003).
[43] M. Caputo, J. Roy. Astronom. Soc. 13, 529 (1967).

[44] G. Jumarie, Comput. Math. Appl. 51, 1367 (2006).

[45] R. Khalil, M.A. Horani, A. Yousef, M. Sababheh, J. Comput. Appl. Math. 264, 65 (2014).

[46] T. Abdeljawad, J. Computat. Appl. Math. 279, 57 (2015).

[47] A. Atangana, D. Baleanu, A. Alsaedi, Open Math. 13, 889 (2015).

[48] O.S. Iyiola, G.O. Oja, Pramana J. Phys. 85, 567 (2015).

[49] Y. Cenesiz, A. Kurt, Acta Univ. Sapient. Math. 7, 130 (2015).

[50] M. Eslami, H. Rezazadeh, Calcolo 53, 475 (2016).

[51] K. Hosseini, Z. Ayati, Nonlinear Sci. Lett. A 7, 58 (2016).

[52] Y. Cenesiz, D. Baleanu, A. Kurt, O. Tasbozan, Waves Random Compl. Media 27, 103 (2017).

[53] D. Zhao, M. Luo, Calcolo 54, 903 (2017).

[54] Y. Hu, J.H. He, Therm. Sci. 20, 773 (2016).

[55] X.E. Wu, Y.S. Liang, Nonlin. Sci. Lett. A 8, 77 (2017).

[56] H.Y. Liu, Z.M. Li, F.K. Ko, Thermal Sci. 21, 1861 (2017).

[57] J.H. He, S.K. Elegan, Z.B. Li, Phys. Lett. A $\mathbf{3 7 6}$, 257 (2012).

[58] M. Wang, X. Li, J. Zhang, Phys. Lett. A 372, 417 (2008).

[59] A. Bekir, Phys. Lett. A 372, 3400 (2008).

[60] A. Bekir, O. Guner, E. Aksoy, Y. Pandir, AIP Conf. Proc. 1648, 730001 (2015).

[61] H. Triki, A.H. Kara, A. Biswas, Ind. J. Phys. 88 751 (2014).

[62] R. Abazari, S. Jamshidzadeh, A. Biswas, Complexity 21, 151 (2016).

[63] H. Abdel-Gawad, A. Biswas, Acta Phys. Pol. B 47, 1101 (2016).

[64] A. Biswas, A.H. Kara, L. Moraru, H. Triki, S.P. Moshokoa, Proc. Roman. Acad. Series A $\mathbf{1 8}$, 144 (2017).

[65] A. Biswas, M. Ekici, A. Sonmezoglu, Nonlin. Anal. Modell. Control 23, 942 (2018).

[66] H. Kheiri, A. Jabbari, A. Yildirim, A.K. Alomari, World J. Modell. Simulat. 9, 3 (2013). 